\title{
Neural network analysis of clinical variables predicts escalated care in COVID-19 patients: a retrospective study
}

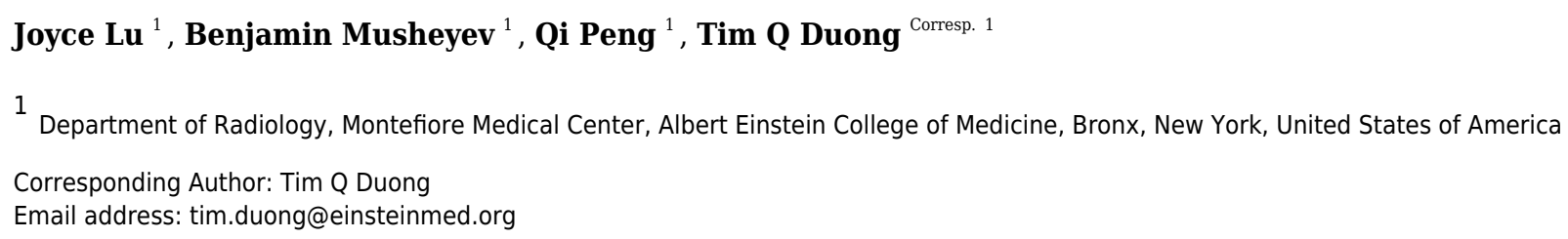

This study sought to identify the most important clinical variables that can be used to determine which COVID-19 patients hospitalized in the general floor will need escalated care early on using neural networks. Analysis was performed on hospitalized COVID-19 patients between February 7, 2020 and May 4, 2020 in Stony Brook Hospital.

Demographics, comorbidities, laboratory tests, vital signs, and blood gases were collected. We compared those data obtained at the time in emergency department and the time of intensive care unit (ICU) upgrade of: i) COVID-19 patients admitted to the general floor $(\mathrm{N}=1203)$ versus those directly admitted to ICU $(\mathrm{N}=104)$, and ii) patients not upgraded to ICU $(\mathrm{N}=979)$ versus those upgraded to the ICU $(\mathrm{N}=224)$ from the general floor. A neural network algorithm was used to predict ICU admission, with $80 \%$ training and $20 \%$ testing. Prediction performance used area under the curve (AUC) of the receiver operating characteristic analysis (ROC). We found that C-reactive protein, lactate dehydrogenase, creatinine, white-blood cell count, D-dimer, and lymphocyte count showed temporal divergence between COVID-19 patients hospitalized in the general floor that were upgraded to ICU compared to those that were not. The neural network predictive model essentially ranked the same laboratory variables to be important predictors of needing ICU care. The AUC for predicting ICU admission was $0.782 \pm 0.013$ for the test dataset. Adding vital sign and blood-gas data improved AUC $(0.822 \pm 0.018)$. This work could help frontline physicians to anticipate downstream ICU need to more effectively allocate healthcare resources. 
1 Neural network analysis of clinical variables predicts escalated care in COVID-19 patients: a retrospective study

3

4

5

$6{ }^{1}$ Department of Radiology, Montefiore Medical Center, Albert Einstein College of Medicine, Bronx, New

7 York

8

$9 *$ Tim.duong@einsteinmed.org

10 Word Count for Text: 2800

11

12 


\section{ABSTRACT}

14

15

This study sought to identify the most important clinical variables that can be used to determine which COVID-19 patients hospitalized in the general floor will need escalated care early on using neural networks. Analysis was performed on hospitalized COVID-19 patients between February 7, 2020 and May 4, 2020 in Stony Brook Hospital. Demographics, comorbidities, laboratory tests, vital signs, and blood gases were collected. We compared those data obtained at the time in emergency department and the time of intensive care unit (ICU) upgrade of: i) COVID-19 patients admitted to the general floor ( $\mathrm{N}=1203$ ) versus those directly admitted to ICU $(\mathrm{N}=104)$, and ii) patients not upgraded to ICU $(\mathrm{N}=979)$ versus those upgraded to the ICU $(\mathrm{N}=224)$ from the general floor. A neural network algorithm was used to predict ICU admission, with $80 \%$ training and 20\% testing. Prediction performance used area under the curve (AUC) of the receiver operating characteristic analysis (ROC). We found that C-reactive protein, lactate dehydrogenase, creatinine, white-blood cell count, D-dimer, and lymphocyte count showed temporal divergence between COVID-19 patients hospitalized in the general floor that were upgraded to ICU compared to those that were not. The neural network predictive model essentially ranked the same laboratory variables to be important predictors of needing ICU care. The AUC for predicting ICU admission was $0.782 \pm 0.013$ for the test dataset. Adding vital sign and blood-gas data improved AUC (0.822 \pm 0.018$)$. This work could help frontline physicians to anticipate downstream ICU need to more effectively allocate healthcare resources.

Key words: Machine learning, coronavirus, pneumonia, SARS-CoV-2, prediction model. 


\section{INTRODUCTION}

Since it was first reported in Wuhan, China in December 2019 (Huang et al. 2020; Li et al. 2020a; Zhu et al. 2020b), the coronavirus disease 2019 (COVID-19) has infected over 27 million people and killed more than 880,000 people worldwide (September 6, 2020). There are recent spikes in COVID-19 cases and there will likely be second waves in many countries. To date, it is challenging for emergency room physicians to objectively and reliably determine which patients need escalated care (i.e., ICU admission) or anticipate ICU needs downstream for effective allocation of healthcare resources in part because much is still unknown about this disease.

Many studies have reported the use of patient demographics, clinical presentations, comorbidities, vital sign data, and laboratory blood tests to predict in-hospital outcomes (see reviews (Brown et al. 2020; Rodriguez-Morales et al. 2020; Wynants et al. 2020)). Some earlier studies found that: i) age and CRP thresholds are good predictor of mortality ( $\mathrm{Lu}$ et al. 2020), ii) age, lymphocyte count, $\mathrm{LDH}$ and $\mathrm{SpO}_{2}$ are independent predictors of mortality (Xie et al. 2020, in press), iii) comorbidity, older age, lower lymphocyte and higher LDH at presentation to be independent high-risk factors for COVID-19 progression (Ji et al. 2020), iv) mildly elevated alanine aminotransferase, myalgias, and hemoglobin at presentation to be predictive of severe acute respiratory distress syndrome (ARDS) of COVID- 19 with $70 \%$ to $80 \%$ accuracy (Jiang et al. 2020), and v) lactate dehydrogenase, procalcitonin, pulse oxygen saturation, smoking history, and lymphocyte count were predictive of ICU admission, and heart failure, procalcitonin, lactate dehydrogenase, chronic obstructive pulmonary disease, pulse oxygen saturation, heart rate, and age were predictive of mortality (Zhao et al. 2020). These studies have relatively small sample sizes.

Most of these published studies to predict outcomes associated COVID-19 used logistic regression. Machine learning (ML) is increasingly being used in medicine (Deo 2015; Hwang et al. 2019; Santos et al. 2019). ML uses computer algorithms to learn relationships amongst different data elements to relate to outcomes without the need to specify the exact relationship amongst these data elements to outcome variables. ML is ideally suited for analyzing large number of data elements. Machine learning methods outperform humans in many tasks in medicine (Killock 2020). With increasing computing power and big data, ML is expected to play an important role in medicine. A neural network (NN), in particular, is an artificial neural network with multiple layers between the input and output layers. NNs are based on algorithms inspired from the biological structure and functioning of a brain to aid machines with intelligence, consisting of neurons, synapses, weights, biases. A few studies have used machine learning to predict inhospital outcomes associated with COVID-19 (Hou et al. 2021, in press; Li et al. 2020b; Yan et al. 2020, in press; Yuan et al. 2020; Zhu et al. 2020a).

Although some predictors of mortality and critical illnesses were shared amongst these studies, there is currently no consensus as to which clinical variables are most predictive of mortality or the needs 
71 for escalated care. Moreover, these prior studies predicted critical illness and mortality using data obtained

72 at admission to the emergency department. COVID-19 patients came into the emergency department at

73 various disease severity. We argue that it is more relevant to study hospitalized COVID-19 patients in the

74 general floor who were subsequently upgraded to ICU to identify the clinical variables that predict escalated 75 care.

Thus, the goal of this study was to identify the clinical variables that can be used to determine which patients hospitalized COVID-19 patients in the general floor will need to be upgraded to ICU early on by comparing between those not upgraded to the ICU from the general floor versus those subsequently upgraded to the ICU. Clinical variables were obtained at the time of arrival to the emergency department and at the time of ICU upgrade. For references, we also compared between COVID-19 patients admitted to the general floor versus those immediately admitted to ICU. As a secondary analysis, we employed a simple neural-network algorithm to these data to identify and predict the most important clinical variables that informed the need for escalated care.

\section{MATERIAL AND METHODS}

Study population and data collection: This study is approved by Stony Brook University Institutional Review Board. Our IRB ID number was IRB2020-00207. Our IRB waived the need for informed consent from patients in the study. This retrospective study utilized the COVID-19 Persons Under Investigation (PUI) registry (N=6,678) of the Stony Brook Hospital ED from February 7, 2020 to June 30, 2020. There were 2,892 COVID-19 positive patients as determined by real-time polymerase chain reaction (RT-PCR) for severe acute respiratory syndrome coronavirus 2 (SARS-CoV-2), of which 1430 were hospitalized. Patients who were $<18$ years old, still in the hospital at the time of analysis, and did not have full codes were excluded.

The final sample sizes included 1203 patients admitted to general floor (“general floor”, Group A) and 104 directly admitted to the ICU from the ED ("direct ICU”, Group B), 979 patients remained on the general floor ("no upgrade", Group C) and 224 were upgraded from the general floor to the ICU ("upgrade ICU”, Group D) (Figure 1).

Demographic information, chronic comorbidities, laboratory tests, vital signs and blood gases were collected. Demographics included age, gender, ethnicity and race. Chronic comorbidities included smoking, diabetes, hypertension, asthma, chronic obstructive pulmonary disease (COPD), coronary artery disease, heart failure, cancer, immunosuppression and chronic kidney disease. Laboratory tests included C-reactive protein (CRP), D-dimer, ferritin, lactate dehydrogenase (LDH), white blood cell count (WBC), lymphocytes count (lymph), procalcitonin (procal), alanine aminotransferase (ALT), aspartate transaminase (AST), brain natriuretic peptide (BNP), creatinine (Cr), and troponin (TNT). Vital signs included heart rate 
105 (HR), respiratory rate (RR), pulse oxygen saturation $\left(\mathrm{SpO}_{2}\right)$, systolic blood pressure (SBP), diastolic blood

106

107

108

109

110

111

112

113

114

115

116

117

118

119

120

121

122

123

124

125

126

127

128

129

130

131

132

133

134

135

136

137

138 pressure (DBP) and temperature (temp). Blood gas variables and others include $\mathrm{pH}, \mathrm{pO}_{2}, \mathrm{pCO}_{2}$, bicarbonate, sodium, hematocrit (HCRIT), and potassium.

These clinical variables were collected for general floor admission (group A) versus direct ICU (group B) at ED admission. Data were collected for the no-upgrade versus upgraded group at ED admission to the general floor. Data were also collected one day prior to ICU upgrade (group D) or three days after hospitalization for the no-upgrade group (Group C). The " $3^{\text {rd }}$ day" was chosen for comparison because the median day for patients to be upgraded to the ICU from the general floor was 3 days.

Preprocessing and neural network prediction model: Bicarbonates, $\mathrm{pCO}_{2}, \mathrm{pO}_{2}, \mathrm{pH}$, hematocrit and troponin were not used in the machine learning analysis because invasive blood gas samples and troponin were not routinely obtained in our hospital on general floor patients. For the rest of the laboratory variables, missing data $(<25 \%)$ were imputated using standard methods (van Buuren \& Groothuis-Oudshoorn 2011).

We employed a simple neural network with two fully connected dense layers using Jupyter Notebook, Tensorflow, and Keras (Figure 2). Only two dense layers were used to avoid overfitting given large number of variables and small sample sizes. Two neural network sequential models were built: one using 11 laboratory tests (excluding vitals and blood gases) and the other using laboratory tests, vitals and blood gases (total 18). The inputs consisted of the clinical variables for no-ICU versus ICU patients: namely those of Group A (floor) at ED admission and Group C (no upgrade) at the corresponding time of upgrade versus Group B (direct ICU) at ED admission and Group D (upgrade) at the time of upgrade. The output was ICU admission, which was binary. For both, the dataset was randomly split into $80 \%$ training data and $20 \%$ testing data. The first dense layer consisted of 4 nodes, and the second dense layer had 3 nodes, both using the rectified linear activation function (ReLU). A Softmax function for activation in the output layer was used. Training was performed for 50 epochs with a batch size of 6 . For the model using laboratory tests, a learning rate of 0.001 was used, whereas for the model using laboratory tests, vitals, and blood gases, a learning rate of 0.0009 proved optimal. We found that these smaller learning rates resulted in the highest accuracy. The model was compiled using the Adam optimizer, an efficient gradient descent algorithm. The clinical variables were ranked using SHAP (SHapley Additive exPlanations), a Python package that explains the output of machine learning models based on game theory. SHAP explains the importance of a feature by calculating the contribution of each feature to the prediction. Specifically, it uses the KernelExplainer to build a weighted linear regression based on the model's predictions and the actual values from the data. It then computes Shapley values from coalitional game theory to determine the variable's importance. Lastly, we calculated cutoffs of the top 6 predictive clinical variables using a nonparametric kernel method to maximize the summation of sensitivity and specificity (Fluss et al. 2005). 
140 Statistical analysis and performance evaluation: Statistical analysis was performed using SPSS v26 (IBM,

141 Armonk, NY) and SAS v9.4 (SAS Institute, Cary, NC). Group comparisons of categorical variables in

142 frequencies and percentages were performed using the Chi-squared test or Fisher exact test. Group 143 comparison of continuous variables in medians and interquartile ranges (IQR) used the Mann-Whitney U

144 test. For all analyses, a $\mathrm{p}$ value $<0.05$ was considered to be statistically significant.

145 For performance evaluation of neural network, data were split $80 \%$ for training and $20 \%$ for testing.

146 Prediction performance was evaluated by area under the curve (AUC) of the receiver operating 147 characteristic (ROC) curve for the test data set. The average ROC curve and AUC were obtained with ten 148 runs and standard deviations were obtained. A p value $<0.05$ was taken to be statistically significant unless 149 otherwise specified.

150

\section{RESULTS}

152

Table 1A summarizes the demographics and comorbidities for the general floor (group A, N=1203)

153

154

155

156

157

158

159

160

161

162

163

164

165

166

167

168

169

170

171 versus direct ICU (group B, N=104). Compared to the general floor group, the direct ICU group had more males $(p=0.005)$, smokers $(p=0.008)$, diabetics $(p=0.047)$ and patients with heart failure $(p=0.016)$. Age, ethnicity, race, and prevalence of hypertension, asthmas, COPD, coronary artery disease, cancer immunosuppression and chronic kidney disease were not statistically different between groups ( $\mathrm{p}>0.05)$.

Table 1B summarizes the demographics and comorbidities for the no-upgrade (group C, N=979) versus upgrade group (group $\mathrm{D}, \mathrm{N}=224$ ). Compared to the no upgrade group, the upgrade ICU group had more males $(\mathrm{p}=0.005)$, and patients with asthma $(\mathrm{p}=0.008)$ but fewer patients with cancer $(\mathrm{p}=0.004)$. Race was different between groups. Age, ethnicity, and prevalence of smoking, hypertension, diabetes, COPD, coronary artery disease, heart failure immunosuppression and chronic kidney disease were not statistically different between groups $(\mathrm{p}>0.05)$.

\section{Laboratory tests:}

Figure 3 plots the laboratory tests for general floor (group A) versus direct ICU (group B) at ED admission, and no-upgrade (group C) versus upgrade (group D) at ED admission and the time of upgrade. WBC, LDH, CRP, TNT, and ferritin were significantly different between the general floor and the direct ICU group at ED admission (red bars). Lymph, WBC, LDH, CRP, AST, CRT, ferritin, and ALT were significantly different between the no-upgrade and upgrade group at the time of admission to the hospital (green bars). Lymph, WBC, and CRP were significantly different between the no-upgrade and upgrade group at the day prior to upgrade (blue bars). 
172

173

174

175

176

177

178

179

180

181

182

183

184

185

186

187

188

189

190

191

192

193

194

195

196

197

198

199

200

201

202

203

204

205

Table 2 integrates the comparison across different groups of Figure 3. LDH, CRP and ferritin were significantly different for: i) the general floor versus direct ICU group at ED admission time point, ii) noupgrade versus upgrade group at ED admission time point, and iii) no-upgrade versus upgrade group at upgrade time point (Table 2, row 1-3). WBC stood out in that it was different for the general floor versus direct ICU group at ED admission, the no-upgrade versus upgrade at upgrade, but it was not different for the no-upgrade versus upgrade at ED admission time point. WBC and CRP significantly decreased in the no-upgrade group (Table $3,4^{\text {th }}$ row). WBC, $\mathrm{LDH}$, and $\mathrm{Cr}$ increased while lymph decreased in the upgrade group (Table $3,5^{\text {th }}$ row).

Lymph, WBC, D-dimer, LDH, CRP, and Cr improved or did not deteriorate between the two time points in the no-upgrade group but deteriorated in the upgrade group (Table $3,6^{\text {th }}$ row). These findings suggest that some of these clinical variables are informative of COVID-19 patients hospitalized in the general floor will need escalated care early on.

\section{Vitals and blood gases:}

Figure 4 plots the vital signs and blood gases for general floor versus direct ICU at ED admission, and no-upgrade versus upgrade at $\mathrm{ED}$ admission and one day prior to upgrade. $\mathrm{RR}, \mathrm{SpO}_{2}$, temperature, $\mathrm{pO}_{2}$, and $\mathrm{pH}$, were significantly different between the general floor versus direct ICU group (red bars). RR, HR, $\mathrm{SpO}_{2}$, temperature, $\mathrm{pH}$, and $\mathrm{pCO}_{2}$ were significantly different between the no-upgrade versus upgrade group (green bars) at the time of admission to hospital. $\mathrm{HR}, \mathrm{SpO}_{2}, \mathrm{DBP}, \mathrm{SDP}$, and temperature were significantly different between the no-upgrade versus upgrade group (blue bars) at the day prior to upgrade.

Table 3 integrates the comparison across different groups in Figure 4. HR, $\mathrm{SpO}_{2}$, and temperature were significantly different for: i) the general floor versus direct ICU group at ED admission time point, ii) no-upgrade versus upgrade at ED admission time point, and iii) no-upgrade versus upgrade at upgrade time point (Table 3, row 1-3). pH stood out in that it was different for the general floor versus direct ICU group at ED admission, no-upgrade versus upgrade at upgrade but it was not different for no-upgrade versus upgrade at ED admission.

For the no upgrade group, RR, HR, DBP, SBP significantly decreased and $\mathrm{SpO}_{2}$ and temperature increased (Table $3,4^{\text {th }}$ row), whereas for the upgrade group, HR and temperature decreased and $\mathrm{SpO}_{2}$ increased (Table $3,5^{\text {th }}$ row). Unlike the laboratory tests, none of the vitals and blood gases showed improvement in the no-upgrade group and deterioration in the upgrade group between the two time points (Table $3,6^{\text {th }}$ row). These findings suggest that some of these clinical variables are informative of COVID19 patients hospitalized in the general floor will need escalated care early on.

\section{Predictors of ICU upgrade}


The neural network model built using laboratory tests ranked CRP, LDH, Cr, WBC, D-dimer, and lymph (in order of importance) to be the top predictors of ICU admission. This model yielded an accuracy of $86 \pm 5 \%$, sensitivity of 0.242 , specificity of 0.966 and AUC of $0.782 \pm 0.013$ for the testing dataset (Figure 5). Note that high specificity and low sensitivity were due to sample asymmetry in which patients of ICU upgrades were fewer than those not upgraded.

The neural network model built using laboratory tests, vitals and blood gases ranked RR, LDH, CRP, DBP, procal, WBC, D-dimer, and $\mathrm{O}_{2}$ (in order of importance) to be the top predictors of ICU admission. This model yielded an accuracy of $88 \pm 7 \%$, sensitivity of 0.364 , specificity of 0.924 , and an AUC of $0.822 \pm 0.018$ for the testing dataset (Figure 6). Note that high specificity and low sensitivity were due to sample asymmetry in which patients of ICU upgrades were fewer than those not upgraded. Cutoffs for the top predictive variables using the training set were determined to be $49.8 \mathrm{mg} / \mathrm{dL}$ for CRP, 1.05 $\mathrm{mg} / \mathrm{dL}$ for $\mathrm{Cr}, 285 \mathrm{ng} / \mathrm{mL}$ for d-dimer, $392 \mathrm{ng} / \mathrm{mL}$ for $\mathrm{LDH}, 12.8 \%$ for lymph, and $8 \mathrm{x} 10^{3} / \mathrm{mL}$ for WBC.

\section{DISCUSSION}

This study investigated the clinical variables associated with direct ICU admission and upgrade to ICU from the general floor. We found that lymphocyte count, white-blood cell count, D-dimer, lactate dehydrogenase, C-reactive protein, and creatinine (unranked) improved or did not deteriorate with time in patients who were not upgraded to the ICU but deteriorated in patients who were upgraded to the ICU, showing temporal divergence. The learning predictive model using laboratory tests ranked C-reactive protein, lactate dehydrogenase, creatinine, white-blood cell count, D-dimer, and lymphocyte count (in divergence. The performance of the predictive model using these top predictors yielded an AUC of $0.782 \pm 0.013$ for predicting ICU admission on the test dataset. Adding vitals and blood-gas data further improved prediction performance $(0.822 \pm 0.018)$.

230

Compared to the general floor group, the direct ICU group had significantly more males, smokers, diabetics and patients with heart failure. Compared to the no upgrade group, the upgrade ICU group had more males, and patients with asthma but fewer patients with cancer. Smokers, diabetics and patients with heart failure were more likely to receive escalated care at ED admission. Patients with asthma was the only comorbidity that were associated with ICU upgrade. Some major comorbidities were important factor for ICU admission especially at ED admission, but less so for ICU upgrade, suggesting that ED physicians might consider major comorbidities as factor needing escalated care.

\section{Clinical variables associated with ICU admission}


Essentially all the laboratory test results of hospitalized COVID-19 patients were outside of

241

242

243

24

245

246

247

248

249

250

251

252

253

254

255

256

257

258

259

260

261

262

263

264

265

266

267

268

269

270

271

272

273 normative physiologic ranges. The normative ranges of major laboratory tests were: lymphocytes $25-33 \%$, WBC 0.5 to $11.0 \times 10^{9} / \mathrm{L}$, D-dimer $<250 \mathrm{ng} / \mathrm{mL}, \mathrm{LDH} 45-90 \mathrm{U} / \mathrm{L}, \mathrm{CRP}<10 \mathrm{mg} / \mathrm{dL}, \mathrm{AST} 5-40 \mathrm{U} / \mathrm{L}, \mathrm{Cr}$ 0.84 to $1.21 \mathrm{mg} / \mathrm{dL}$, troponin $<0.04 \mathrm{ng} / \mathrm{mL}$, ferritin male: $15-200 \mathrm{ng} / \mathrm{mL}$ (male) and $12-150 \mathrm{ng} / \mathrm{mL}, \mathrm{BNP}$ $<100 \mathrm{pg} / \mathrm{mL}$, procal $<0.15 \mathrm{ng} / \mathrm{mL}$, and ALT $8-40 \mathrm{U} / \mathrm{L}$. Elevated values of these laboratory tests indicate increased disease severity in COVID-19, except lymphocyte count where lower values are associated with worse prognosis (Brown et al. 2020). Note that these clinical variables could be dependent on sex, age, ethnicity and race and are shown here for reference and might not be of use clinically.

Many laboratory tests showed worse disease severity in the direct or upgrade ICU group compared to general floor and no-upgrade group. However, we found that these laboratory tests by themselves were inadequate to reliably determine which patients required ICU admission. Often time, there were no appreciable differences between those directly admitted or upgraded to the ICU and those admitted to the general floor. For example, LDH, CRP and ferritin were significantly different for the general floor versus direct ICU group at ED admission, and no-upgrade versus upgrade group for both ED admission and at time of the ICU upgrade, suggesting they might not be useful to distinguish ICU upgrade despite being abnormal due to COVID-19. WBC stood out in that it was different for the general floor versus direct ICU group at ED admission and the no-upgrade versus upgrade group at the time of upgrade, but not for the noupgrade versus upgrade group at ED admission, suggesting it is one of the most informative variables of ICU upgrade.

Our innovative approach was thus to identify the laboratory tests that showed improvement or plateau between the two time points in the no-upgrade group but deteriorated in the upgrade group. The laboratory tests that showed temporal divergence were identified to be lymphocyte count, white-blood cell count, D-dimer, lactate dehydrogenase, C-reactive protein, and creatinine (unranked). By contrast, most vitals and blood gases did not show such temporal divergence between groups, suggesting that vital signs and blood gases might be overall less important when compared to laboratory tests. This appears counter intuitive because vitals are readily available and are often informative in emergency room situation. Possible explanations are: i) $\mathrm{SpO}_{2}$ might be affected by supplemental oxygen inhalation, ii) RR, HR, SBP and DBP could be highly variables, iii) these vital signs were within normal normative physiological ranges although there were group differences. We concluded that vital signs and blood gases appear to be overall less informative in predicting ICU admission compared to laboratory tests.

\section{Neural Network analysis}

To further explore whether the above-mentioned laboratory variables are predictive of direct and upgrade ICU admission, we developed a neural network model, trained it on $80 \%$ of the data, and tested it 
274 independently on $20 \%$ of data that the model had not seen before. Our neural network model identified $C$ -

275 reactive protein, lactate dehydrogenase, creatinine, white-blood cell count, D-dimer, and lymphocyte count

276 (in orders of importance) to be the top predictors of ICU admission. These variables showed substantial

277 overlaps with those variables exhibiting temporal divergence described above. The performance of the

278 predictive model using these top predictors yielded an AUC of 0.782 for predicting ICU admission from

279 the testing dataset. Note that high specificity and low sensitivity were due to sample asymmetry in which

280 patients of ICU upgrades were fewer than those not upgraded. Adding vital and blood-gas data improved

281 prediction performance, yielding an AUC of 0.822 for predicting ICU admission from the test dataset. It is

282 worth noting that RR was one of the highly ranked variables. This is not surprising because COVID-19

283 patients usually exhibited respiratory distress. Taken together, there is corroborative evidence that a few

284 laboratory tests and vital signs are amongst the most important predictors of severe illness that warrants

285 escalated care.

286

\section{Limitations}

This study has several limitations. This is a retrospective study carried out in a single hospital. As in all observational studies, other residual confounders might exist that were not accounted for in our

290

291

292

293

294

295

296

297

298

299

300

301

302

303

304

305

306 analysis. These findings need to be replicated in a large and multi-institutional setting for generalizability. It might be challenging however to achieve generalizability across hospitals because the COVID-19 pandemic circumstance is unusual and evolving and how we treat COVID-19 patient is also evolving. ICU admission rate could depend on countries, hospital practice, patient loads, available ICU beds, and when during the pandemic the data were collected, amongst others. At the time of our study, our hospital was not limited by available ICU beds. It is conceivable that our model might not work for patients in Wuhan because their patients were more severe, amongst others. Our model also might not work on data from second COVID-19 wave. This is not because the model is wrong, but rather we believe that it is necessary to retrain predictive model with local data. We only explored NN. Alternatively, random forest, Xgboost, kernel support vector machine and other more sophisticated machine learning methods could also be explored. To date it is generally not trivial for hospitals to share clinical COVID-19 data because of lack of infrastructure to do so seamlessly or concerns about patient data privacy, amongst others. There is a national effort to share deidentified clinical COVID-19 data but this is not yet available.

\section{Conclusions}

This study provided corroborative evidence that WBC, lymphocyte count, D-dimer, lactate dehydrogenase, C-reactive protein, and creatinine are amongst the most important predictors of severe 
307 illness requiring ICU care. This work could help frontline physicians to better manage COVID-19 patients 308 by anticipating downstream ICU needs to more effectively allocate healthcare resources. 


\section{REFERENCES}

311

312

313

314

315

316

317

318

319

320

321

322

323

324

325

326

327

328

329

330

331

332

333

334

335

336

337

338

339

340

341

342

343

344

345

346

347

348

349

350

351

352

353

354

355

356

357

358

https://coronavirus.jhu.edu/map.html. Johns Hopkin University.

https://www.merckmanuals.com/professional/ (assessed Aug 31, 2020).

Brown RAC, Barnard J, Harris-Skillman E, Harbinson B, Dunne B, Drake J, Roche S, Harris E, Gunnel J, Frost J, Angus B, and Hodgson S. 2020. Lymphocytopaenia is associated with severe SARSCoV-2 disease: A Systematic Review and Meta-Analysis of Clinical Data. medRxiv:2020.2004.2014.20064659. 10.1101/2020.04.14.20064659

Deo RC. 2015. Machine Learning in Medicine. Circulation 132:1920-1930. 10.1161/CIRCULATIONAHA.115.001593

Fluss R, Faraggi D, and Reiser B. 2005. Estimation of the Youden Index and its associated cutoff point. Biom J 47:458-472. 10.1002/bimj.200410135

Hou H, Zhao Z, Chen A, Li H, and Duong TQ. 2021, in press. Machining learning predicts the need for escalated care and mortality in COVID-19 patients from clinical variables. International $J$ Medical Sciences.

Huang C, Wang Y, Li X, Ren L, Zhao J, Hu Y, Zhang L, Fan G, Xu J, Gu X, Cheng Z, Yu T, Xia J, Wei Y, Wu W, Xie X, Yin W, Li H, Liu M, Xiao Y, Gao H, Guo L, Xie J, Wang G, Jiang R, Gao Z, Jin Q, Wang J, and Cao B. 2020. Clinical features of patients infected with 2019 novel coronavirus in Wuhan, China. Lancet 395:497-506. 10.1016/S0140-6736(20)30183-5

Hwang TJ, Kesselheim AS, and Vokinger KN. 2019. Lifecycle Regulation of Artificial Intelligence- and Machine Learning-Based Software Devices in Medicine. JAMA. 10.1001/jama.2019.16842

Ji D, Zhang D, Xu J, Chen Z, Yang T, Zhao P, Chen G, Cheng G, Wang Y, Bi J, Tan L, Lau G, and Qin E. 2020. Prediction for Progression Risk in Patients with COVID-19 Pneumonia: the CALL Score. Clin Infect Dis. 10.1093/cid/ciaa414

Jiang X, Coffee M, Bari A, Wang J, Jiang X, Huang J, Shi J, Dai J, Cai J, Zhang T, Wu Z, He G, and Huang Y. 2020. Towards an Artificial Intelligence Framework for Data-Driven Prediction of Coronavirus Clinical Severity. Computers, Materials \& Continua 63:537-551.

Killock D. 2020. AI outperforms radiologists in mammographic screening. Nat Rev Clin Oncol 17:134. 10.1038/s41571-020-0329-7

Leung K, Wu JT, Liu D, and Leung GM. 2020. First-wave COVID-19 transmissibility and severity in China outside Hubei after control measures, and second-wave scenario planning: a modelling impact assessment. The Lancet 395:1382-1393. 10.1016/s0140-6736(20)30746-7

Li Q, Guan X, Wu P, Wang X, Zhou L, Tong Y, Ren R, Leung KSM, Lau EHY, Wong JY, Xing X, Xiang N, Wu Y, Li C, Chen Q, Li D, Liu T, Zhao J, Liu M, Tu W, Chen C, Jin L, Yang R, Wang Q, Zhou S, Wang R, Liu H, Luo Y, Liu Y, Shao G, Li H, Tao Z, Yang Y, Deng Z, Liu B, Ma Z, Zhang Y, Shi G, Lam TTY, Wu JT, Gao GF, Cowling BJ, Yang B, Leung GM, and Feng Z. 2020a. Early Transmission Dynamics in Wuhan, China, of Novel Coronavirus-Infected Pneumonia. N Engl J Med 382:1199-1207. 10.1056/NEJMoa2001316

Li X, Ge P, Zhu J, Li H, Graham J, Singer A, Richman PS, and Duong TQ. 2020b. Deep learning prediction of likelihood of ICU admission and mortality in COVID-19 patients using clinical variables. PeerJ 8:e10337. 10.7717/peerj.10337

Lu J, Hu S, Fan R, Liu Z, Yin X, Wang Q, Lv Q, Cai Z, Li H, and Hu Y. 2020. ACP risk grade: a simple mortality index for patients with confirmed or suspected severe acute respiratory syndrome coronavirus 2 disease (COVID-19) during the early stage of outbreak in Wuhan, China. medRxiv.

Rodriguez-Morales AJ, Cardona-Ospina JA, Gutierrez-Ocampo E, Villamizar-Pena R, Holguin-Rivera Y, Escalera-Antezana JP, Alvarado-Arnez LE, Bonilla-Aldana DK, Franco-Paredes C, HenaoMartinez AF, Paniz-Mondolfi A, Lagos-Grisales GJ, Ramirez-Vallejo E, Suarez JA, Zambrano LI, Villamil-Gomez WE, Balbin-Ramon GJ, Rabaan AA, Harapan H, Dhama K, Nishiura H, Kataoka H, Ahmad T, Sah R, and Latin American Network of Coronavirus Disease C-REahwlo. 
2020. Clinical, laboratory and imaging features of COVID-19: A systematic review and metaanalysis. Travel Med Infect Dis 34:101623. 10.1016/j.tmaid.2020.101623

Santos MK, Ferreira Junior JR, Wada DT, Tenorio APM, Barbosa MHN, and Marques PMA. 2019. Artificial intelligence, machine learning, computer-aided diagnosis, and radiomics: advances in imaging towards to precision medicine. Radiol Bras 52:387-396. 10.1590/0100-3984.2019.0049 van Buuren S, and Groothuis-Oudshoorn K. 2011. mice: Multivariate Imputation by Chained Equations in R. Journal of Statistical Software 45:1-67.

Wynants L, Van Calster B, Collins GS, Riley RD, Heinze G, Schuit E, Bonten MMJ, Damen JAA, Debray TPA, De Vos M, Dhiman P, Haller MC, Harhay MO, Henckaerts L, Kreuzberger N, Lohman A, Luijken K, Ma J, Andaur CL, Reitsma JB, Sergeant JC, Shi C, Skoetz N, Smits LJM, Snell KIE, Sperrin M, Spijker R, Steyerberg EW, Takada T, van Kuijk SMJ, van Royen FS, Wallisch C, Hooft L, Moons KGM, and van Smeden M. 2020. Prediction models for diagnosis and prognosis of covid-19 infection: systematic review and critical appraisal. BMJ 369:m1328. 10.1136/bmj.m1328

Xie J, Hungerford D, Chen H, Abrams ST, Li S, Li X, Tong Z, Du B, Qiu H, and Toh CH. 2020, in press. Development and external validation of a prognostic multivariable model on admission for hospitalized patients with COVID-19. medRxiv.

Yan L, Zhang HT, Goncalves J, Xiao Y, Wang M, Guo Y, Sun C, Tang X, Jing L, Zhang M, Huang X, Xiao Y, Cao H, Chen Y, Ren T, Wang F, Xiao Y, Huang S, Tan X, Huang N, Jiao B, Cheng C, Zhang Y, Luo A, Mombaerts L, J. J, and Cao Z. 2020, in press. An interpretable mortality prediction model for COVID-19 patients. Nat Machine Intelligence.

Yuan Y, Sun C, Tang X, Cheng C, Mombaerts L, Wang M, Hu T, Sun C, Guo Y, Li X, Xu H, Ren T, Xiao Y, Xiao Y, Zhu H, Chen C, Liu Y, Liang Z, Cao Z, Zhang HT, Paschaldis IC, Liu Q, Gonalves J, Zhong Q, and Yan L. 2020. Development and validation of a prognostic risk score system for COVID-19 inpatients: A multi-center retrospective study in China. Pre-print. 10.21203/rs.3.rs-41151/v1

Zhao Z, Chen A, Hou W, Graham JM, Li H, Richman PS, Thode HC, Singer AJ, and Duong TQ. 2020. Prediction model and risk scores of ICU admission and mortality in COVID-19. PLoS One 15:e0236618. 10.1371/journal.pone.0236618

Zhu JS, Ge P, Jiang C, Zhang Y, Li X, Zhao Z, Zhang L, and Duong TQ. 2020a. Deep-learning artificial intelligence analysis of clinical variables predicts mortality in COVID-19 patients. $J$ Am Coll Emerg Physicians Open 1:1364-1373. 10.1002/emp2.12205

Zhu N, Zhang D, Wang W, Li X, Yang B, Song J, Zhao X, Huang B, Shi W, Lu R, Niu P, Zhan F, Ma X, Wang D, Xu W, Wu G, Gao GF, Tan W, China Novel Coronavirus I, and Research T. 2020b. A Novel Coronavirus from Patients with Pneumonia in China, 2019. N Engl J Med 382:727-733. 10.1056/NEJMoa2001017 


\section{Author contributions statements}

398

$399 \mathrm{JL}$ - collected data, analyzed data, drafted paper

$400 \mathrm{BM}-$ analyzed data and drafted paper

401 QP - edited paper

402 TD - supervised, wrote paper

403

404 Competing interest statement

405

406 The author declared no competing interests.

407

408

409 Funding

410 None 


\section{Figure legends}

413

414

Figure 1. Patient selection flowchart. The final sample sizes included 1203 patients admitted to general

415 floor ("general floor", Group A) and 104 directly admitted to the ICU from the ED ("direct ICU", Group B), 979 patients remained on the general floor ("no upgrade", Group C) and 224 were upgraded from the general floor to the ICU (“upgrade ICU”, Group D).

418

Figure 2. Architecture of both neural networks. The model consists of one input layer, one output layer with two fully connected hidden layers.

421

422 Figure 3. Laboratory tests for group A (floor) and B (direct ICU) at ED admission, and group C (no 423 upgrade) and group D (upgrade) at two time points (at ED admission and one day prior upgrade and 424 equivalence). SI conversion factors: To convert alanine aminotransferase and lactate dehydrogenase to 425 microkatal per liter, multiply by 0.0167 ; C-reactive protein to milligram per liter, multiply by 10 ; D-dimer 426 to nanomole per liter, multiply by 0.0054 ; leukocytes to $\times 10^{9}$ per liter, multiply by 0.001 . Error bars are 427 SEM. ${ }^{*} \mathrm{p}<0.05, * * \mathrm{p}<0.01, * * * \mathrm{p}<.005$. Sample sizes for each bar graphs are shown. Note that a lower 428 lymphocyte count, whereas higher values of the other laboratory variables, are associated with worse 429 prognosis.

430

431 Figure 4. Vital signs and blood gases for group A (floor) and B (direct ICU) at ED admission, and group $432 \mathrm{C}$ (no upgrade) and group D (upgrade) at two time points (at ED admission and one day prior upgrade and 433 equivalence). Error bars are SEM. ${ }^{*} \mathrm{p}<0.05, * * \mathrm{p}<0.01, * * * \mathrm{p}<.005$. Sample sizes for each bar graphs are 434 shown.

Figure 5. AUC of neural network model built using laboratory tests.

Figure 6. AUC of neural network model built using laboratory tests, vitals, and blood gases. 


\section{Table $\mathbf{1}$ (on next page)}

1A) Laboratory tests, vital signs, and blood gases of patients

i) admitted to general floor from the emergency department ("floor") and ii) admitted to ICU directly from emergency department ("direct ICU"). 1B) Laboratory tests, vital signs, and blood gases of patients: i) not upgraded ("no-upgrade"), and ii) upgrade to ICU from general floor ("upgrade"). Group comparison of categorical variables in frequencies and percentages used $\chi^{2}$ test or Fisher exact tests. Group comparison of continuous variables in medians and interquartile ranges (IQR) used the Mann-Whitney $U$ test. Abbreviation: COPD, chronic obstructive pulmonary disease. IQR, interquartile range. $\mathrm{SpO}_{2}, \mathrm{O}_{2}$, oxygen saturation. 
1 Table 1A. Laboratory tests, vital signs, and blood gases of patients: i) admitted to general floor from the emergency department ("floor") and ii) admitted to ICU directly from emergency department ("direct ICU”).

\begin{tabular}{|c|c|c|c|}
\hline & $\begin{array}{c}\text { Floor } \\
\mathrm{N}=1203\end{array}$ & $\begin{array}{c}\text { Direct ICU } \\
\mathrm{N}=104\end{array}$ & $\mathrm{P}$ value \\
\hline \multicolumn{4}{|l|}{ Demographics } \\
\hline Age, median (IQR) & $60(49,73)$ & $63(52,74)$ & 0.529 \\
\hline Sex & & & 0.005 \\
\hline Male & $687(57.1 \%)$ & $74(71.2 \%)$ & \\
\hline Female & $516(42.9 \%)$ & $30(28 . \%)$ & \\
\hline Ethnicity & & & 0.175 \\
\hline Hispanic/Latino & $333(27.7 \%)$ & $20(19.2 \%)$ & \\
\hline Non-Hispanic/Latino & $710(59 \%)$ & $69(66.3 \%)$ & \\
\hline Unknown & $160(13.3 \%)$ & $15(14.4 \%)$ & \\
\hline Race & & & 0.784 \\
\hline Caucasian & $629(52.3 \%)$ & $57(54.8 \%)$ & \\
\hline African American & $90(7.5 \%)$ & $7(6.7 \%)$ & \\
\hline Asian & $42(3.5 \%)$ & $5(4.8 \%)$ & \\
\hline American Indian/Alaska Native & $3(0.2 \%)$ & $1(1.0 \%)$ & \\
\hline Pacific Islander & $1(0.1 \%)$ & 0 & \\
\hline More Than One Race & $7(0.6 \%)$ & 0 & \\
\hline Unknown/Not Reported & $431(35.8 \%)$ & $34(32.7 \%)$ & \\
\hline \multicolumn{4}{|l|}{ Comorbidities } \\
\hline Smoking History & & & 0.008 \\
\hline Current Smoker & $49(4.1 \%)$ & $8(7.7 \%)$ & \\
\hline Former Smoker & $250(20.8 \%)$ & $24(23.1 \%)$ & \\
\hline Never Smoker & $839(69.7 \%)$ & $59(56.7 \%)$ & \\
\hline Unknown & $64(5.3 \%)$ & $12(11.5 \%)$ & \\
\hline Diabetes & $309(25.7 \%)$ & $36(34.6 \%)$ & 0.047 \\
\hline Hypertension & $573(47.6 \%)$ & $55(52.9 \%)$ & 0.304 \\
\hline Asthma & $81(6.7 \%)$ & $6(5.8 \%)$ & 0.705 \\
\hline COPD & $96(8.0 \%)$ & $10(9.6 \%)$ & 0.558 \\
\hline Coronary artery disease & $167(13.9 \%)$ & $15(14.4 \%)$ & 0.878 \\
\hline Heart failure & $84(7.0 \%)$ & $14(13.5 \%)$ & 0.016 \\
\hline Cancer & $108(9.0 \%)$ & $11(10.6 \%)$ & 0.586 \\
\hline Immunosuppression & $91(7.6 \%)$ & $9(8.7 \%)$ & 0.688 \\
\hline Chronic kidney disease & $112(9.3 \%)$ & $12(11.5 \%)$ & 0.457 \\
\hline
\end{tabular}

4

5

6

7

8

9

10

11

12

13

14 
15

16 Table 1B. Laboratory tests, vital signs, and blood gases of patients: i) not upgraded ("no-upgrade"), and ii) upgrade to ICU from general floor ("upgrade").

\begin{tabular}{|c|c|c|c|}
\hline & $\begin{array}{c}\text { No-upgrade } \\
\mathrm{N}=979\end{array}$ & $\begin{array}{c}\text { Upgrade } \\
\mathrm{N}=224\end{array}$ & $\mathrm{P}$ value \\
\hline \multicolumn{4}{|l|}{ Demographics } \\
\hline Age, median (IQR) & $60(49,72)$ & $60(50,70)$ & 0.307 \\
\hline Sex & & & $<0.001$ \\
\hline Male & $534(54.5 \%)$ & $153(68.3 \%)$ & \\
\hline Female & $445(45.5 \%)$ & $71(31.7 \%)$ & \\
\hline Ethnicity & & & 0.056 \\
\hline Hispanic/Latino & $226(21.2 \%)$ & $67(29.9 \%)$ & \\
\hline Non-Hispanic/Latino & $589(60.2 \%)$ & $121(54.0 \%)$ & \\
\hline Unknown & $124(12.8 \%)$ & $36(16.0 \%)$ & \\
\hline Race & & & 0.005 \\
\hline Caucasian & $531(54.2 \%)$ & $98(43.8 \%)$ & \\
\hline African American & $76(7.8 \%)$ & $14(6.3 \%)$ & \\
\hline Asian & $27(2.8 \%)$ & $15(6.7 \%)$ & \\
\hline American Indian/Alaska Native & $2(0.2 \%)$ & $1(0.5 \%)$ & \\
\hline Pacific Islander & $1(0.1 \%)$ & 0 & \\
\hline More Than One Race & $7(0.7 \%)$ & 0 & \\
\hline Unknown/Not Reported & $335(34.2 \%)$ & $96(42.9 \%)$ & \\
\hline \multicolumn{4}{|l|}{ Comorbidities } \\
\hline Smoking History & & & 0.247 \\
\hline Current Smoker & $48(4.5 \%)$ & $5(2.2 \%)$ & \\
\hline Former Smoker & $237(22.1 \%)$ & $42(18.7 \%)$ & \\
\hline Never Smoker & $728(67.8 \%)$ & $164(72.9 \%)$ & \\
\hline Unknown & $60(5.6 \%)$ & $14(6.2 \%)$ & \\
\hline Diabetes & $246(25.1 \%)$ & $63(28.1 \%)$ & 0.354 \\
\hline Hypertension & $464(47.4 \%)$ & $109(46.7 \%)$ & 0.732 \\
\hline Asthma & $57(5.8 \%)$ & $24(10.7 \%)$ & 0.008 \\
\hline COPD & $84(8.6 \%)$ & $12(5.4 \%)$ & 0.108 \\
\hline Coronary artery disease & $132(13.5 \%)$ & $35(15.6 \%)$ & 0.403 \\
\hline Heart failure & $68(7.0 \%)$ & $16(7.1 \%)$ & 0.917 \\
\hline Cancer & $99(10.1 \%)$ & $9(4.0 \%)$ & 0.004 \\
\hline Immunosuppression & $77(7.9 \%)$ & $14(6.3 \%)$ & 0.410 \\
\hline Chronic kidney disease & $94(9.6 \%)$ & $18(8.0 \%)$ & 0.467 \\
\hline
\end{tabular}




\section{Table 2 (on next page)}

Comparison of laboratory tests. This is a summary of the results in Figure 3. 
1 Table 2. Comparison of laboratory tests. This is a summary of the results in Figure 3. Note that at upgrade 2 means 1 day prior to upgrade, $\uparrow=$ significant increase where $p<0.05$, $\uparrow \uparrow=$ significant increase where $p$ $3<0.01, \uparrow \uparrow \uparrow=$ significant increase where $p<0.005 . \downarrow=$ significant decrease where $p<0.05, \downarrow \downarrow=$ 4 significant decrease where $p<0.01, \downarrow \downarrow \downarrow=$ significant decrease where $p<0.005$. X: denotes variables that 5 showed group $\mathrm{C}$ improved or plateaued, but group D deteriorated between two time points.

6

\begin{tabular}{|c|c|c|c|c|c|c|c|c|c|c|c|c|c|}
\hline Row & & lymh & WBC & D-dimer & LDH & CRP & Fer & $\mathbf{C r}$ & TNT & AST & BNP & Procal & ALT \\
\hline 1 & Group A vs B at admission & & 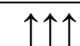 & & $\uparrow \uparrow \uparrow \uparrow$ & $\uparrow \uparrow \uparrow \uparrow$ & $\uparrow$ & & $\downarrow \downarrow \downarrow$ & & & & \\
\hline 2 & Group C vs D at admission & $\downarrow \downarrow \downarrow$ & & & $\uparrow \uparrow \uparrow$ & $\uparrow \uparrow \uparrow$ & 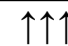 & & & $\uparrow$ & & & \\
\hline 3 & Group C vs D at upgrade & $\downarrow \downarrow \downarrow$ & $\uparrow \uparrow \uparrow$ & $\uparrow$ & $\uparrow \uparrow \uparrow$ & $\uparrow \uparrow \uparrow$ & $\uparrow \uparrow \uparrow$ & $\uparrow$ & & $\uparrow \uparrow$ & & & $\uparrow$ \\
\hline 4 & at admission vs at upgrade for $\mathrm{C}$ & & $\downarrow \downarrow \downarrow$ & & & $\downarrow \downarrow \downarrow$ & & & & & & & \\
\hline 5 & at admission vs at upgrade for $\mathrm{D}$ & $\downarrow \downarrow \downarrow$ & 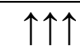 & $\uparrow$ & 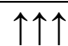 & & & 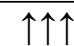 & & & & & \\
\hline 6 & $\begin{array}{l}\text { C improved or plateau } \\
\text { but D deteriorated }\end{array}$ & $\mathbf{X}$ & $\mathbf{X}$ & $\mathbf{X}$ & $\mathbf{X}$ & $\mathbf{X}$ & & $\mathbf{X}$ & & & & & \\
\hline
\end{tabular}

7 


\section{Table 3 (on next page)}

Comparisons for vitals and blood gases. This is a summary of the results in Figure 4 
1 Table 3. Comparisons for vitals and blood gases. This is a summary of the results in Figure 4. Note that at 2 upgrade means 1 day prior to upgrade, $\uparrow=$ significant increase where $p<0.05$, $\uparrow \uparrow=$ significant increase 3 where $p<0.01$, $\uparrow \uparrow \uparrow=$ significant increase where $p<0.005 . \downarrow=$ significant decrease where $p<0.05$, $\downarrow \downarrow$ $4=$ significant decrease where $p<0.01$, $\downarrow \downarrow \downarrow=$ significant decrease where $p<0.005$. X: denotes variables 5 that showed $\mathrm{C}$ improved or plateaued, but $\mathrm{D}$ deteriorated between two time points

6

\begin{tabular}{|c|c|c|c|c|c|c|c|c|c|c|c|c|c|c|c|}
\hline $\begin{array}{l}\text { Ro } \\
\text { W }\end{array}$ & & $\mathbf{R R}$ & HR & $\mathrm{SpO}_{2}$ & DBP & SBP & Temp & $\mathbf{p O}_{2}$ & pH & $\mathbf{p C O}_{2}$ & Herit & $\begin{array}{l}\text { Blood } \\
\text { Bicarb }\end{array}$ & $\begin{array}{l}\text { Serum } \\
\text { Bicarb }\end{array}$ & $\mathbf{K}^{+}$ & $\mathbf{N a}^{+}$ \\
\hline 1 & Group A vs B at admission & $\uparrow \uparrow \uparrow$ & & $\downarrow \downarrow \downarrow$ & & & $\downarrow$ & $\uparrow \uparrow \uparrow$ & $\downarrow \downarrow \downarrow$ & & & $\downarrow$ & $\downarrow \downarrow \downarrow$ & & \\
\hline 2 & Group C vs D at admission & $\uparrow \uparrow \uparrow \uparrow$ & & $\downarrow \downarrow \downarrow$ & & $\downarrow \downarrow \downarrow$ & $\uparrow \uparrow \uparrow \uparrow$ & & & & & & $\downarrow \downarrow \downarrow$ & & $\downarrow \downarrow$ \\
\hline 3 & Group C vs D at upgrade & 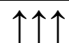 & $\uparrow \uparrow \uparrow$ & $\downarrow \downarrow \downarrow$ & & & 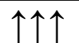 & & $\downarrow$ & $\uparrow$ & $\uparrow$ & & & & $\downarrow \downarrow$ \\
\hline 4 & & & & & & & & & & & & & & & \\
\hline 4 & at admission vs at upgrade for $\mathrm{C}$ & $\downarrow \downarrow \downarrow$ & $\downarrow \downarrow \downarrow$ & $\uparrow \uparrow \uparrow$ & $\downarrow \downarrow \downarrow$ & $\downarrow \downarrow \downarrow$ & $\downarrow \downarrow \downarrow$ & & & & $\downarrow \downarrow \downarrow$ & & $\downarrow \downarrow \downarrow$ & & $\uparrow \uparrow \uparrow$ \\
\hline 5 & at admission vs at upgrade for $\mathrm{D}$ & & $\downarrow \downarrow \downarrow$ & $\uparrow \uparrow \uparrow$ & & & $\downarrow \downarrow \downarrow$ & & & & $\downarrow \downarrow \downarrow$ & & $\downarrow$ & & $\uparrow \uparrow \uparrow$ \\
\hline 6 & $\begin{array}{l}\mathrm{C} \text { improved or unchanged } \\
\text { but } \mathrm{D} \text { deteriorated }\end{array}$ & & & & & & & & & & & & & & \\
\hline
\end{tabular}


Figure 1

Patient selection flowchart.

The final sample sizes included 1203 patients admitted to general floor ("general floor", Group A) and 104 directly admitted to the ICU from the ED ("direct ICU", Group B), 979 patients remained on the general floor ("no upgrade", Group C) and 224 were upgraded from the general floor to the ICU ("upgrade ICU", Group D). 


\section{Stony Brook COVID-19 Dataset Summary}

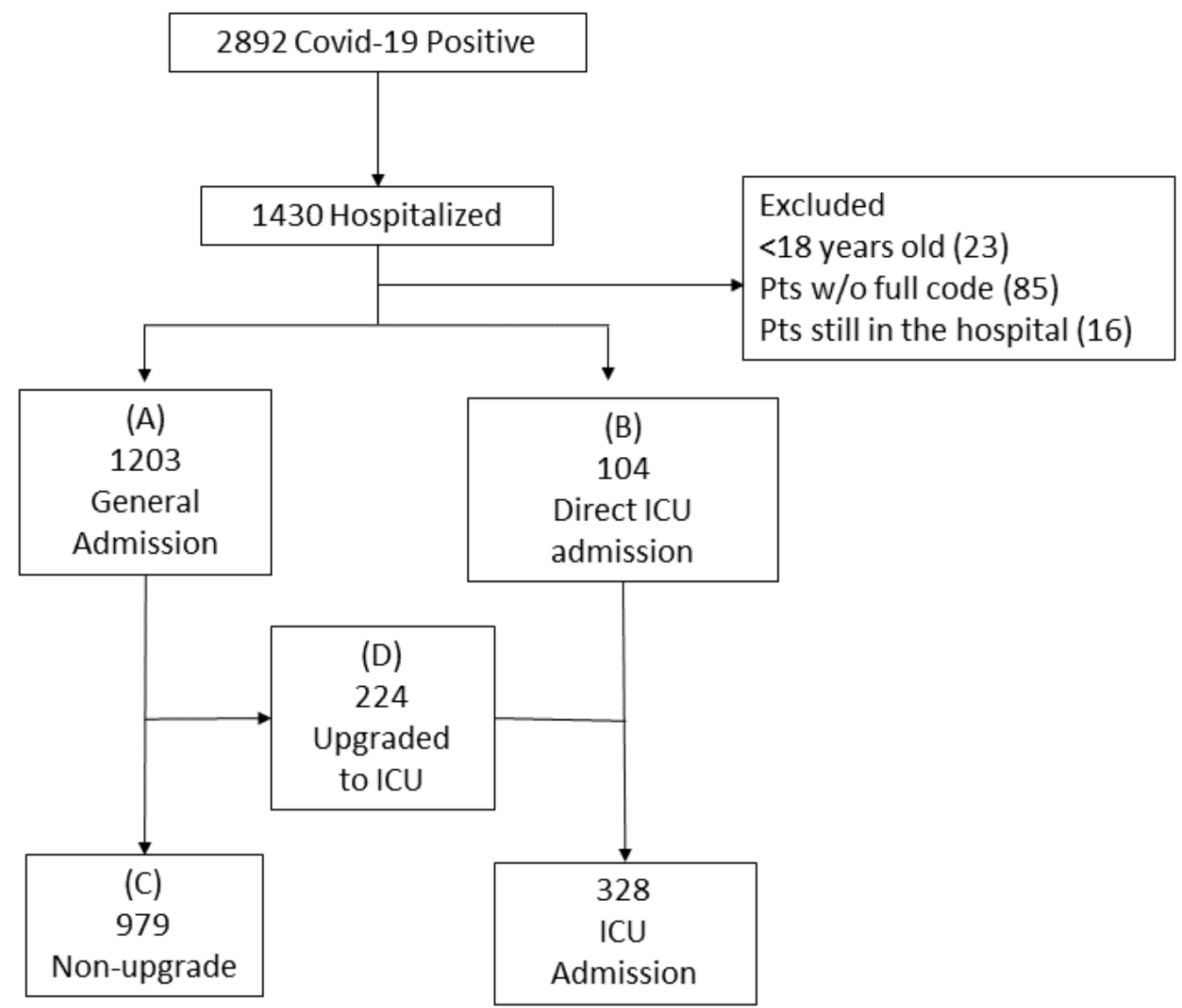


Figure 2

Architecture of both neural networks

The model consists of one input layer, one output layer with two fully connected hidden layers.

Hidden Layer

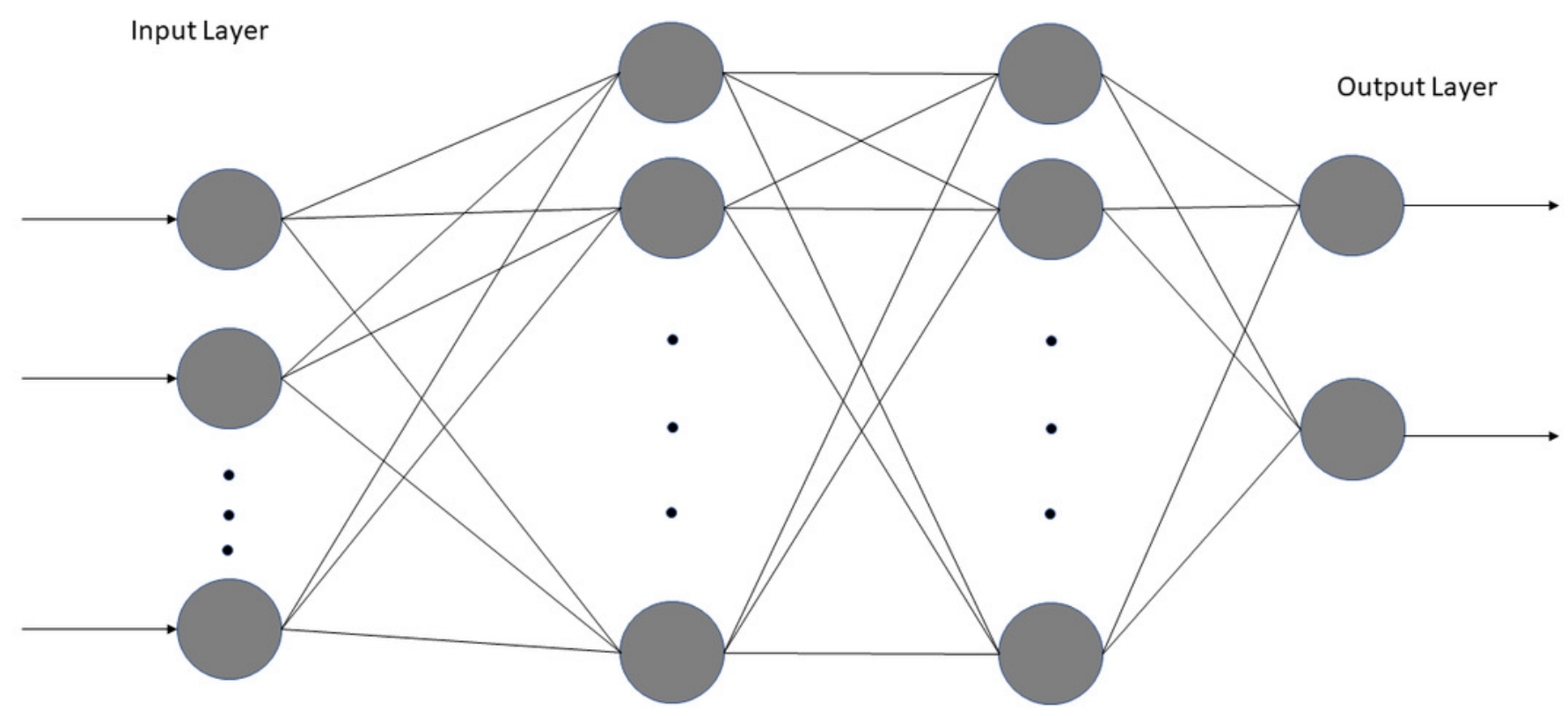




\section{Figure 3}

\section{Laboratory tests}

Laboratory tests for group A (floor) and B (direct ICU) at ED admission, and group C (no upgrade) and group D (upgrade) at two time points (at ED admission and one day prior upgrade and equivalence). SI conversion factors: To convert alanine aminotransferase and lactate dehydrogenase to microkatal per liter, multiply by 0.0167 ; C-reactive protein to milligram per liter, multiply by 10 ; D-dimer to nanomole per liter, multiply by 0.0054 ;

leukocytes to $\times 10^{9}$ per liter, multiply by 0.001 . Error bars are SEM. $* p<0.05, * * p<0.01, * * *$ $p<.005$. Sample sizes for each bar graphs are shown. Note that a lower lymphocyte count, whereas higher values of the other laboratory variables, are associated with worse prognosis. 


\section{Laboratory Tests}
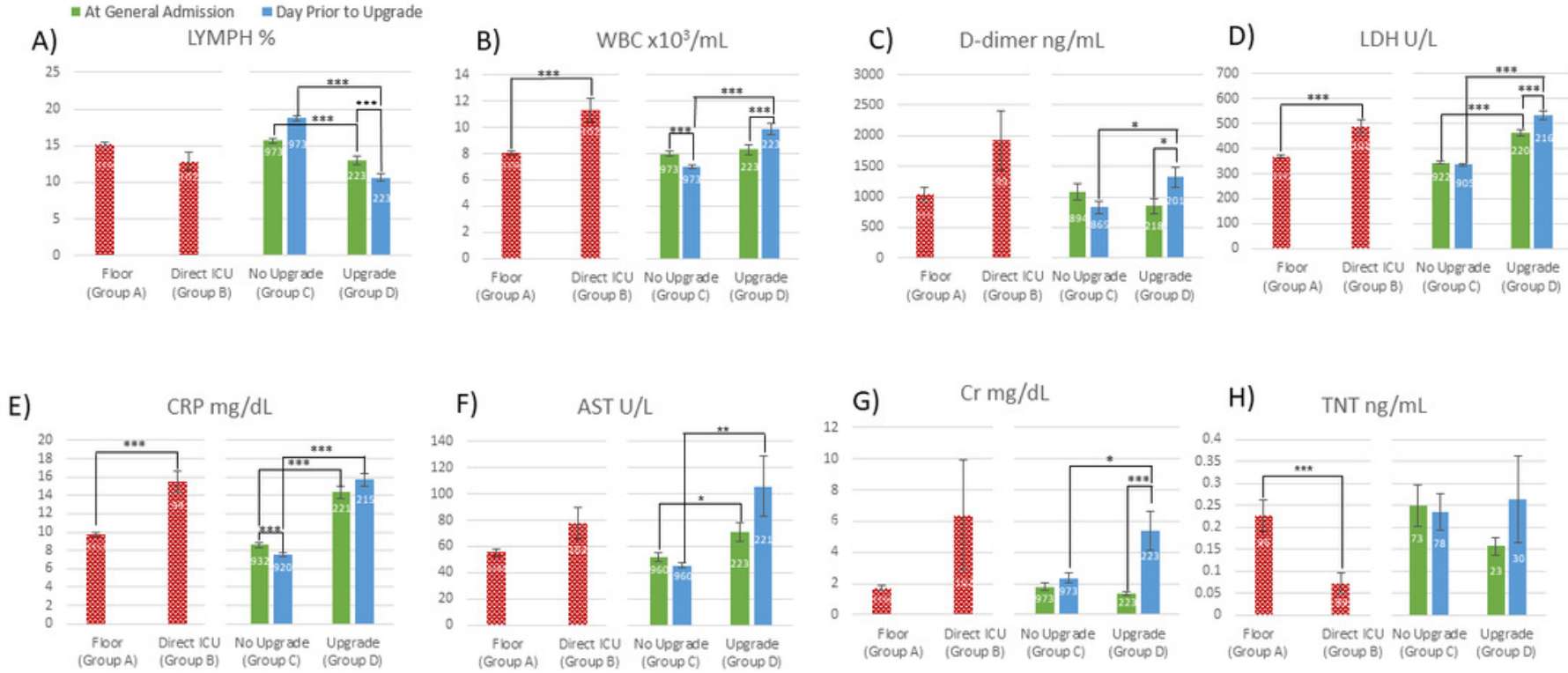

H) $\quad$ TNT ng/mL
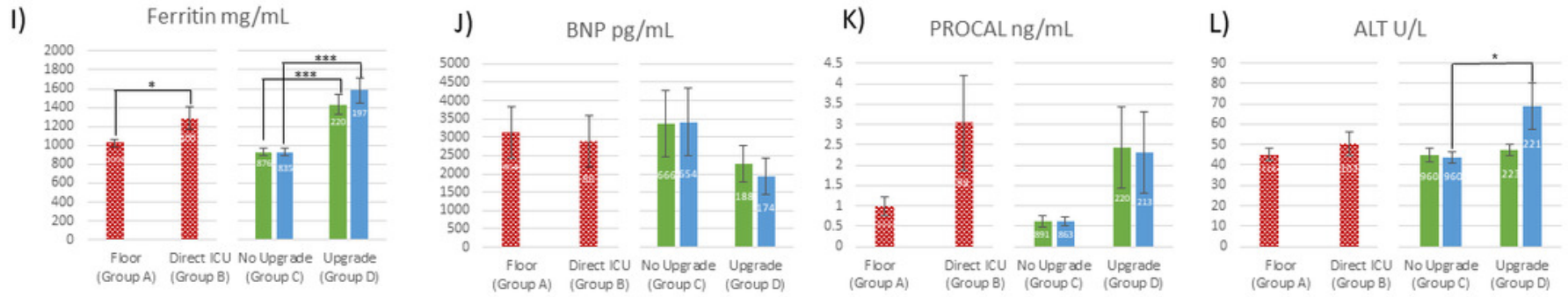
Figure 4

Vital signs and blood gases

Vital signs and blood gases for group A (floor) and B (direct ICU) at ED admission, and group $C$ (no upgrade) and group D (upgrade) at two time points (at ED admission and one day prior upgrade and equivalence). Error bars are SEM. $* p<0.05, * * p<0.01, * * * p<.005$. Sample sizes for each bar graphs are shown. 
= At General Admission $=$ Day Prior to Upgrade

A)
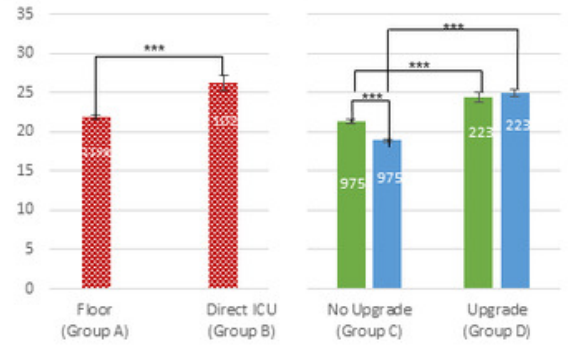

D)
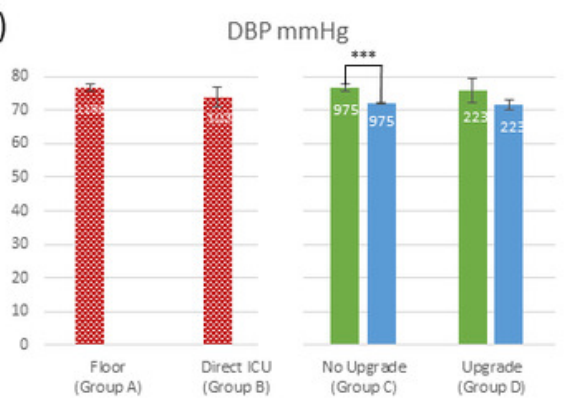
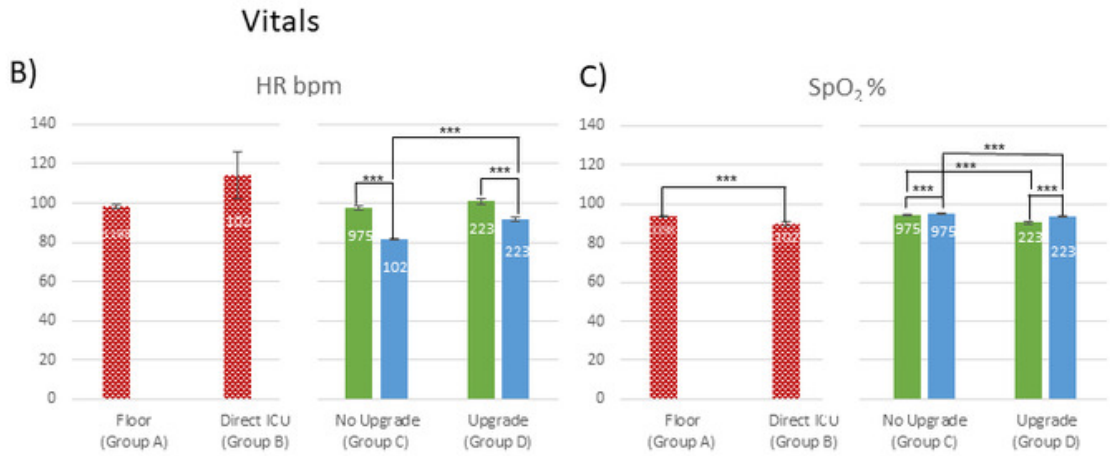

E)

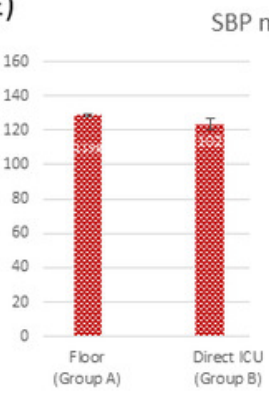

$\mathrm{SBP} \mathrm{mmHg}$

F)
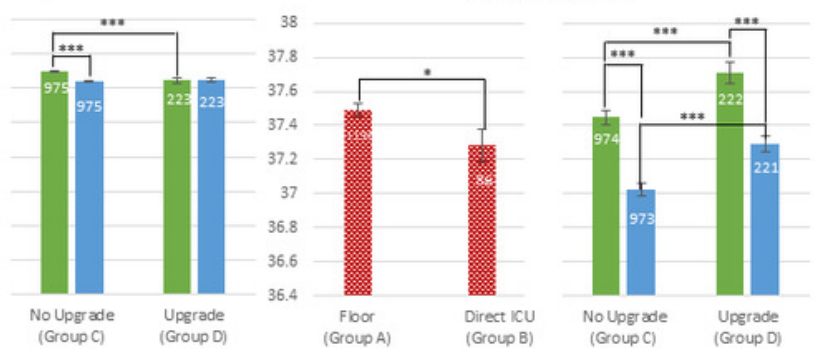

Blood Gas and others

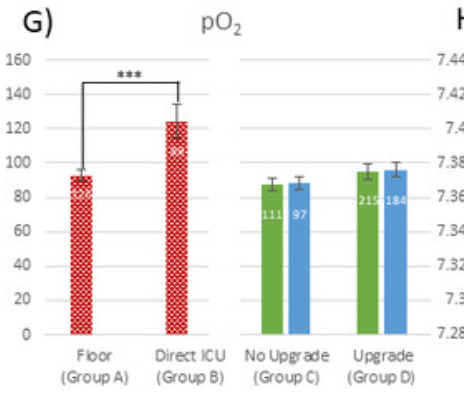

H)

$\mathrm{pH}$

I)

$\mathrm{pCO}_{2}$

J) BICARB (arterial blood) mEq/L
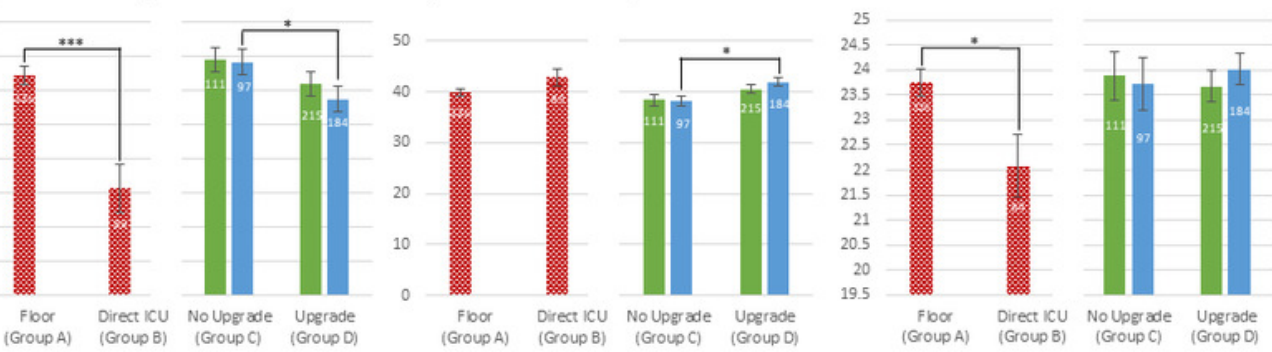

K)

L)

M)

HCRIT\%

BICARB (serum) $\mathrm{mEq} / \mathrm{L}$

$\mathrm{K}^{+} \mathrm{mEq} / \mathrm{L}$

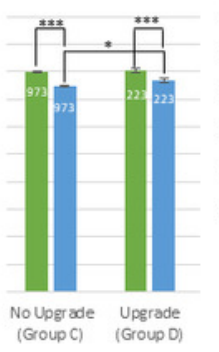

4.5

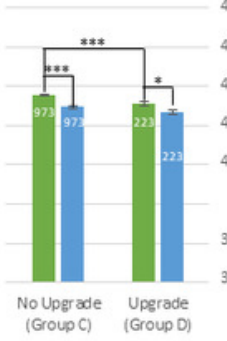

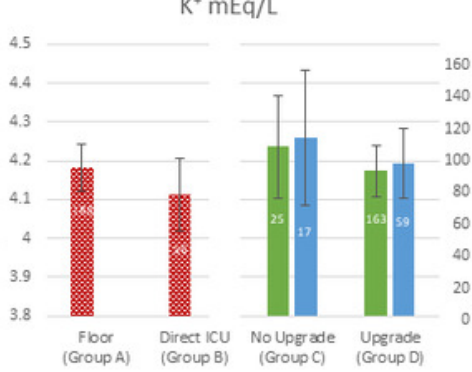

N)

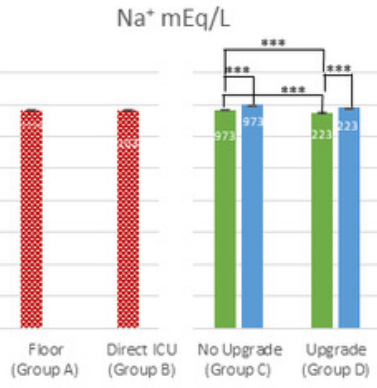


Figure 5

AUC of deep neural network model built using laboratory tests.

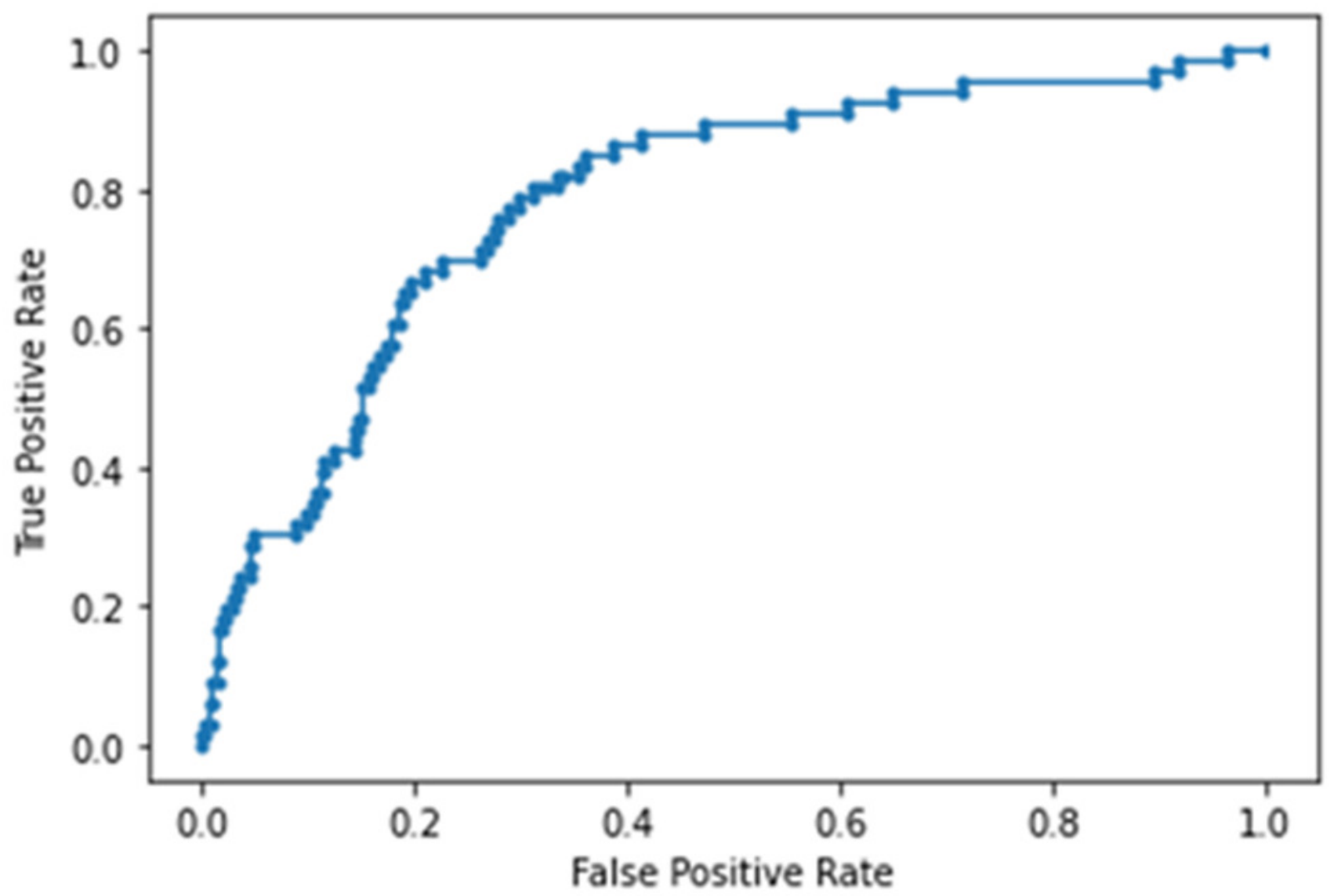


Figure 6

AUC of deep neural network model built using laboratory tests, vitals, and blood gases.

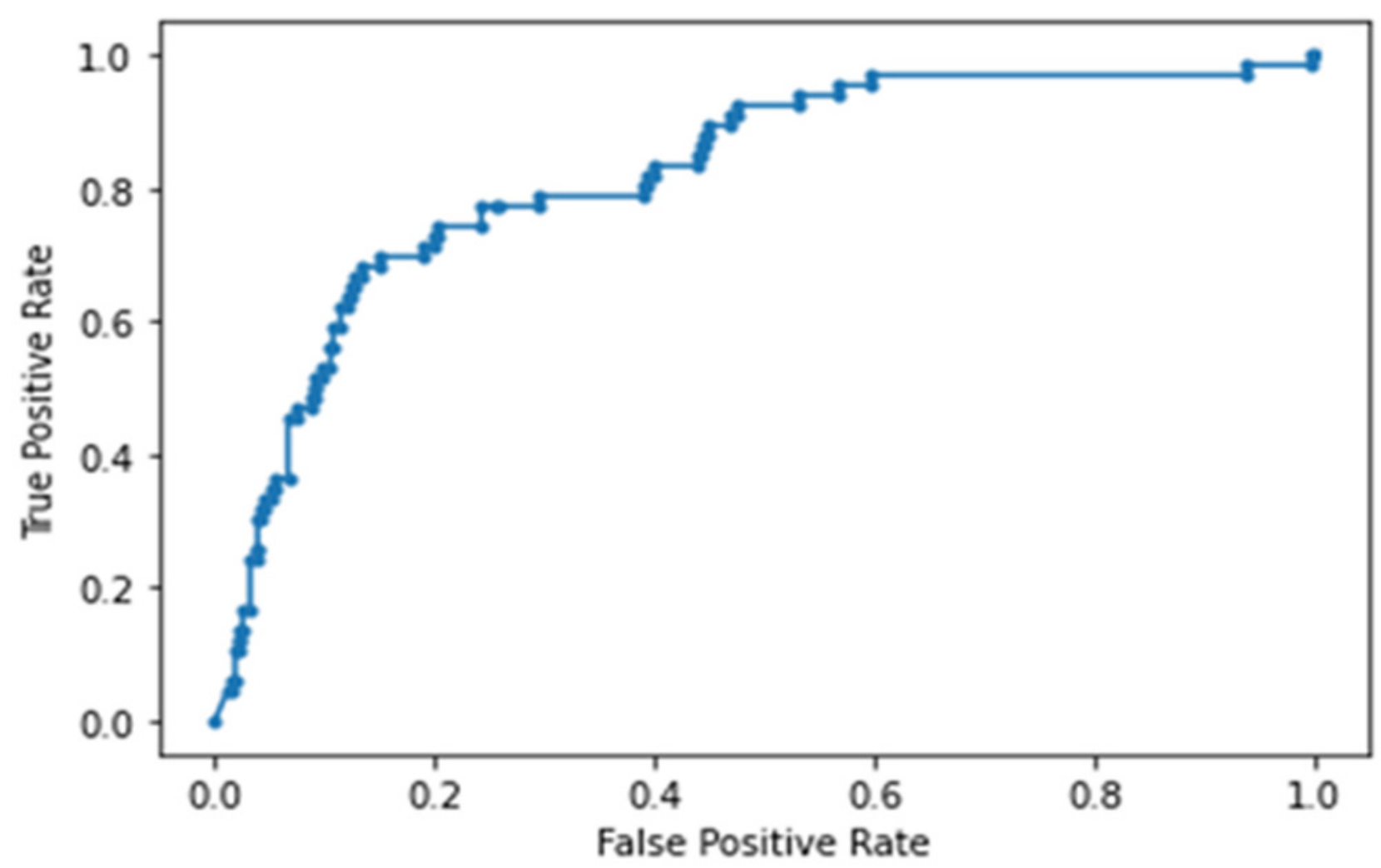

